tions and MRSA/VRE cultured from enteral feeding tubes used in the same neonatal intensive care unit during the same time period but in different neonates. DNA fingerprinting then was used to compare MRSA and VRE cultured from feeding tubes with MRSA/VRE isolates cultured from clinical infections.

There were $23 S$. aureus isolates; 12 of 23 were methicillin resistant (MRSA). There were 4 MRSA infections in patients without feeding tubes. DNA fingerprinting showed that the MRSA species causing each of the clinical infections also was in the feeding tubes of other infants. There were no VRE infections during the study period.

The authors concluded that feeding tubes are a reservoir for antibiotic-resistant pathogens that can be transmitted to other infants.

FROM: Mehall JR, Kite CA, Gilliam CH, Jackson RJ, Smith SD. Enteral feeding tubes are a reservoir for nosocomial antibiotic-resistant pathogens. J Pediatr Surg 2002;37:1011-1012.

\section{Intranasal Mupirocin to Prevent Postoperative Staphylococcus aureus Infections}

Patients with nasal carriage of Staphylococcus aureus have an increased risk of surgical-site infections caused by that organism. Treatment with mupirocin ointment can reduce the rate of nasal carriage and may prevent postoperative $S$. aureus infections. Perl, from Johns Hopkins, Baltimore, Maryland, and co-investigators, from the University of Iowa Colleges of Medicine and Public Health, Iowa City, conducted a randomized, double-blind, placebo-controlled trial to determine whether intranasal treatment with mupirocin reduces the rate of $S$. aureus infections at surgical sites and prevents other nosocomial infections.

Of 4,030 enrolled patients who underwent general, gynecologic, neurologic, or cardiothoracic surgery, 3,864 were included in the intention-to-treat analysis. Overall, $2.3 \%$ of mupirocin recipients and $2.4 \%$ of placebo recipients had $S$. aureus infections at surgical sites. Of the 891 patients ( $23.1 \%$ of the 3,864 who completed the study) who had $S$. aureus in their anterior nares, 444 received mupirocin and 447 received placebo. Among the patients with nasal carriage of $S$. aureus, $4.0 \%$ of those who received mupirocin had nosocomial $S$. aureus infections, as compared with $7.7 \%$ of those who received placebo (odds ratio for infection, $0.49 ; 95 \%$ confidence interval, 0.25 to $0.92 ; P=$ $.02)$.

The authors concluded that prophylactic intranasal application of mupirocin did not significantly reduce the rate of $S$. aureus surgical-site infections overall, but it did significantly decrease the rate of all nosocomial $S$. aureus infections among the patients who were $S$. aureus carriers.

FROM: Perl TM, Cullen JJ, Wenzel RP, et al. Intranasal mupirocin to prevent postoperative Staphylococcus aureus infections. N Engl J Med 2002;346:1871-1877.

\section{Risk of Airborne Transmission of MRSA in an Otolaryngology Surgery Unit}

Shiomori and colleagues from the University of Occupational and Environmental Health, School of Medicine, Kitakyushu, Japan, conducted a study to quantitatively investigate the existence of airborne methicillin-resistant Staphylococcus aureus (MRSA) in a hospital environment. They performed phenotyping and genotyping of MRSA isolates to study MRSA epidemiology. They also performed prospective surveillance of patients with MRSA infections or colonization. Air samples were taken by an air sampler; samples were also obtained from object surfaces. An epidemiologic study of MRSA isolates was performed with an antibiotic susceptibility test, coagulase typing, and pulsed-field gel electrophoresis. The study was conducted in three single-patient rooms in a 37-bed otolaryngology-head and neck surgery unit. Three patients with squamous cell head and neck cancer were observed to have been colonized or infected with MRSA after surgery.

The MRSA samples were collected from the air in single-patient rooms during both a period of rest and when bed sheets were being changed. Isolates of MRSA were detected in all stages (from stage $1[>7 \mu \mathrm{m}]$ to stage 6 [0.65 to $1.1 \mu \mathrm{m}]$ ). Approximately $20 \%$ of the MRSA particles were within a respirable range of less than $4 \mu \mathrm{m}$. MRSA was also isolated from inanimate objects, such as sinks, floors, and bed sheets, in the rooms of the patients with MRSA infections and from the patients' hands. An epidemiologic study demonstrated that clinical isolates of MRSA in the patient ward were of one origin and that the isolates from the air and from inanimate objects were identical to the MRSA strains that caused infection or colonization in the patients.

The authors concluded that MRSA was recirculated among the patients, the air, and the inanimate objects, especially when there was movement in the rooms. Airborne MRSA may play a role in MRSA colonization in the nasal cavity or in respiratory tract MRSA infections. Measures should be taken to prevent the spread of airborne MRSA to control nosocomial MRSA infection in hospitals.

FROM: Shiomori T, Miyamoto $\mathrm{H}$, Makishima $\mathrm{K}$. Significance of airborne transmission of methicillin-resistant Staphylococcus aureus in an otolaryngology-head and neck surgery unit. Arch Otolaryngol Head Neck Surg 2001;127:644-648.

\section{Nosocomial Outbreak of Fluoroquinolone- Resistant Salmonella Infection}

Infection with fluoroquinolone-resistant strains of salmonella is rare, as is nosocomial salmonella infection. Olsen and colleagues from the Division of Bacterial and Mycotic Diseases, Centers for Disease Control and Prevention, Atlanta, Georgia, describe the first recognized outbreak of fluoroquinolone-resistant salmonella infections in the United States, which occurred in two nursing homes and one hospital in Oregon. They interviewed medical staff and reviewed patients' charts and death certificates. In 